\title{
Influence of lactoferrin in preventing preterm delivery: A pilot study
}

\author{
GIULIANA GIUNTA $^{1}$, LORENA GIUFFRIDA ${ }^{1}$, KATIA MANGANO $^{2}$, PAOLO FAGONE $^{2}$ and ANTONIO CIANCI $^{1}$ \\ ${ }^{1}$ Department of Maternal-Infant and Radiological Sciences, University Hospital G. Rodolico; \\ ${ }^{2}$ Department of Bio-medical Sciences, University of Catania, Catania, Italy
}

Received April 8, 2011; Accepted August 22, 2011

DOI: $10.3892 / \mathrm{mmr} .2011 .584$

\begin{abstract}
Lactoferrin ( $\mathrm{Lf}$ ) is an approximately 80-kDa ironbinding glycoprotein, belonging to the transferrin family, with well-known bacteriostatic and bactericidal properties. It is produced and stored in specific (secondary) neutrophil granules and released during neutrophil activation and degranulation. Nowadays, Lf has a well-known therapeutic indication for combating iron deficiency anemia (IDA) in pregnant women. Studies suggest that Lf plays an important role against cervicovaginal infections by decreasing cytokines levels, such as interleukin (IL)-6, in cervicovaginal fluid. The aim of this preliminary trial was to evaluate the effectiveness of Lf in preventing preterm delivery caused by cervical infections and ripening. From November 2009 to August 2010, 21 pregnant women (26-32 weeks pregnant), aged between 22 and 36 years, suffering from IDA, at risk of preterm delivery, were prospectively enrolled in the study. One group $(\mathrm{N}=14)$ received $100 \mathrm{mg}$ of recombinant human lactoferrin (rhLf) (lattoferrina; AG-pharma $\left.{ }^{\circledR}\right)$ twice a day before meals, for one month. The other group $(\mathrm{N}=7)$ received $520 \mathrm{mg}$ of ferrous sulfate (FerroGrad $^{\circledR}$; Abbott Laboratories, USA) once a day. The patients underwent transvaginal ultrasound to evaluate cervical length and funneling, and vaginal swabs were used to detect infections and cervicovaginal fluid sample collection to determine IL-6 levels. The results showed a correlation between the oral administration of $200 \mathrm{mg}$ of rhLf with both the normalization of vaginal flora (vaginal infection disappearance) and the decrease in IL-6 cervicovaginal fluid levels in women at risk of preterm delivery.
\end{abstract}

\section{Introduction}

Preterm delivery is one of the most important obstetric pathologies, with a high rate of perinatal morbidity and

Correspondence to: Professor Antonio Cianci, Department of Maternal-Infant and Radiological Sciences, University Hospital G. Rodolico, Catania, Italy

E-mail: acianci@unict.it

Key words: lactoferrin, preterm delivery, IL-6 mortality (1). Generally, preterm delivery is induced by infections, such as abnormal vaginal flora (AVF), full and partial bacterial vaginosis (FBV and PBV) and aerobic vaginitis (AV) (2). Infections lead to the inflammatory host response by two different pathways depending on pathogenic microorganisms. Gram-positive bacteria, such as S. aureus, S. agalactiae and $E$. faecalis, enhance the innate immune system response of the host through the peptidoglycan (PGN), the major component of the cell wall. PGN activates Toll-like receptors 2 and 4 (TLR2/ TLR4), type I transmembrane glycoproteins, that increase the expression of multiple inflammatory genes (3). Gram-negative bacteria, such as E. cloacae, activate the inflammatory host response by the bacterial endotoxin lipopolysaccharide (LPS) (4).

Finally, cytokine production, the synthesis of prostaglandins and the activation of metalloproteinases, glycosaminoglycans and collagenases lead to cervical dilatation and funneling, premature rupture of membranes (PROM) and preterm delivery (5-8).

The above pathogenic scenario anticipates that suppression of the inflammatory host response may be useful to prevent preterm labor and delivery.

Lactoferrin (Lf) is an approximately $80-\mathrm{kDa}$ iron-binding glycoprotein, belonging to the transferrin family, with wellknown bacteriostatic and bactericidal properties, that plays a significant role in iron homeostasis. LF is produced and stored in specific (secondary) neutrophil granules and released during neutrophil activation and degranulation (9). It has been detected in secretary fluids, such as milk, tears, saliva, vaginal secretion, seminal fluid, and amniotic fluid. Therefore, it is considered an important component of host defense against microbial infection (10-12).

Nowadays, Lf has a well-known therapeutic indication for combating iron deficiency anemia (IDA) in pregnant women $(13,14)$, which is still a major health problem in pregnant women (15). Values of hemoglobin corresponding to $11 \mathrm{~g} / \mathrm{dl}$ or less define IDA. The preferred treatment of that pathology consists of oral administration of iron as ferrous sulfate. However, ferrous sulfate often fails to exert significant effects on hypoferremia and may also cause adverse effects. Therefore, Lf represents a novel therapeutic alternative to ferrous sulfate for treating IDA $(13,14)$.

Studies on pregnant female mice have suggested that Lf plays a focal role against cervicovaginal infection by 
decreasing cytokine levels, such as interleukin (IL)-6, on cervicovaginal fluid. Yakuwa and Otsuki have evaluated the capacity of Lf in suppressing uterine cervical dilatation and ripening in a rabbit model, in which preterm labor was induced by bacterial endotoxin LPS (16). Recombinant human Lf (rhLf) was administrated to pregnant rabbits as a vaginal suppository. The histological study of cervical tissues of the rabbits showed remarkable loosening of the connective tissue and edema in the control group (without treatment). These results suggest that rhLF inhibits cervical maturation induced by LPS in rabbits (16).

Taken together, these data prompted us to undertake this open-label double-blind pilot trial aimed at evaluating the impact of rhLf on the prevention of preterm delivery.

\section{Materials and methods}

Patients and treatment. From November 2009 to August 2010, 21 women aged between 22 and 36 years, 26-32 weeks pregnant, suffering from IDA, at risk of preterm delivery who were referred to the Maternal-Infant Health and Radiological Sciences Department, University Hospital G. Rodolico, Catania, Italy, were prospectively enrolled in the study. The inclusion criteria were: IDA, early uterine contraction, cervical length of $<2.5 \mathrm{~cm}$ and/or funneling, no previous preterm birth. Women affected by one or more of the following pathologies were excluded from the study: Metrorrhagia, PROM, twin pregnancy, pregnancy pathologies such as gestational diabetes, gestational hypertension, placenta previa, polyhydramnios, intrauterine growth restriction (IUGR), uterine abnormalities and previous conization.

Patients were divided in two groups: One group received $100 \mathrm{mg}$ of rhLf (lattoferrina ${ }^{\circledR}$; AG-pharma) twice a day before meals for one month and the other group $(\mathrm{N}=7)$ received a tablet of $520 \mathrm{mg}$ of ferrous sulfate (Ferro-Grad ${ }^{\circledR}$-Abbott Laboratories, USA) once a day, as suggested by the Italian standard treatments.

Every woman received a tocolytic treatment with atosiban (Tractocile ${ }^{\circledR}$ ), as stated in the International Guidelines. Atosiban is an inhibitor of the hormones, oxytocin and vasopressin, and it is used as an intravenous medication to treat preterm delivery: Pregnancy weeks from 24 to 33, regular labor (at least 4 contractions/30 min) in combination with an affected cervix (i.e., cervix obliterated $>50 \%$ or open $>1.5 \mathrm{~cm}$ or cervix channel $<25 \mathrm{~mm}$ at vaginal ultrasound) or breaking of the water, and normal fetal heart rate.

At enrollment, and after 10 and 30 days of treatment, the patients underwent obstetric transvaginal ultrasound to evaluate cervical length and funneling, vaginal swabs to detect infections, and cervicovaginal fluid sample collection to determine IL-6 levels.

The study protocol was conformed to the ethical guidelines of the 1975 Helsinki Declaration and it was approved by the Institutional Review Board of the department. Written informed consent was obtained from each female patient upon enrollment.

Microbiological analyses. Standard microbiological methods were followed in this study during swab culture. Specimens were collected with the help of serum-coated cotton-tipped

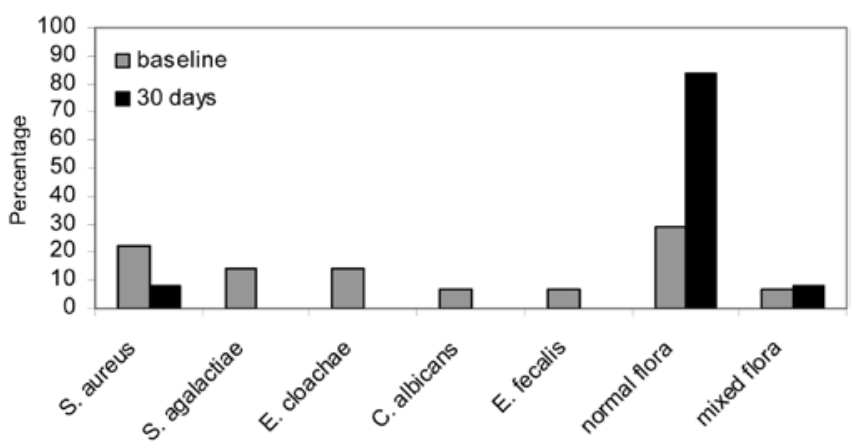

Figure 1. Main pathogens in the vaginal swabs of the LF group at baseline visit and after 30 days of treatment.

swab sticks for culture, following universal precautions and sent for microbiological analysis. Swabs were cultured on 5\% sheep blood agar plates and inoculated into Todd-Hewitt broth for aerobic culture and semiquantitative growth $(2+$ or more was considered to be significant). A vaginal smear was taken to assess vaginal microflora by phase contrast microscopy.

AVF, bacterial vaginosis (BV), AV, increased inflammation (more than ten leucocytes per epithelial cell) and vaginal colonization with Candida spp. were scored according to the standardized classification by Donders, based on the relative quantities of lactobacillary morphotypes present. If lactobacilli of variable size predominate, the flora is considered normal (lactobacillary grade I, LBGI) (2). Lactobacillary grade II is an intermediate flora, with a mixture of lactobacilli and other morphotypes, usually coccoids. Lactobacillary grade III indicates complete disruption of the normal vaginal flora. Flora was defined as normal (NF) when none of the following was present: LBG III, BV, coccoid AV flora, increased vaginal leucocytosis (2).

Sexually transmitted infections caused by organisms, such as Chlamydia trachomatis, Neisseria gonorrhoeae and Trichomonas vaginalis were excluded.

IL-6 determination. IL-6 was determined by sandwich enzyme-linked immunoadsorbent assay (ELISA). The limit of sensitivity of the assay was $1.5 \mathrm{pg} / \mathrm{ml}$. Intra- and inter-assay coefficients of variation were within 5 and $10 \%$, respectively. Those samples with a content of IL- $6<1.5 \mathrm{pg} / \mathrm{ml}$ were assigned this value as a theoretical value to allow statistical analysis.

Outcome was recorded as either preterm birth (PTB; between $26+0$ to 36 weeks +6 days) or birth at term (TB; between $37+0$ and 41 weeks +6 days).

Normally distributed continuous variables were compared using the Student's t-test and stochastic variables were compared using the Chi-square test as appropriate. A value $\mathrm{P}<0.05$ was considered significant.

\section{Results}

At baseline visit, microbiological analysis showed a marked presence of AVF, consisting of predominantly coccoid AV flora, Gram-positive and Gram-negative, and Candida albicans in both groups (Figs. 1 and 2). Women treated with Lf had $71 \%$ of AVF at baseline vs. $71 \%$ of AVF in those treated 

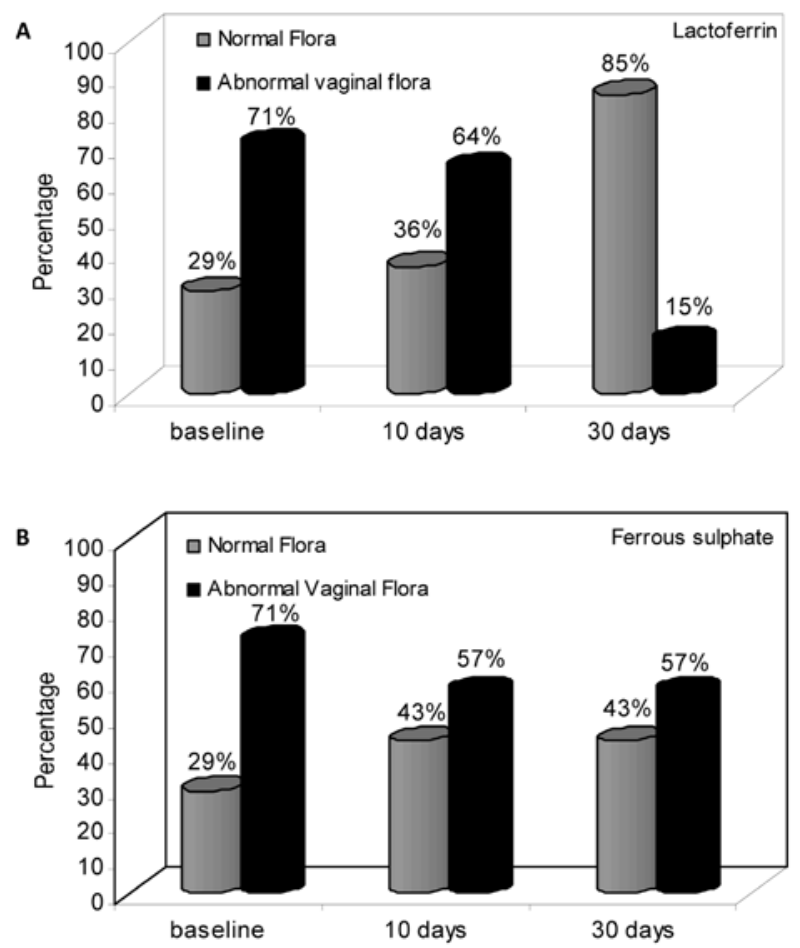

Figure 2. Percentage of normal vaginal flora (NF) and abnormal vaginal flora (AVF) from vaginal swabs of female patients treated with lactoferrin (A) or ferrous sulphate (B) at baseline visit, after 10 days and 30 days of treatment.

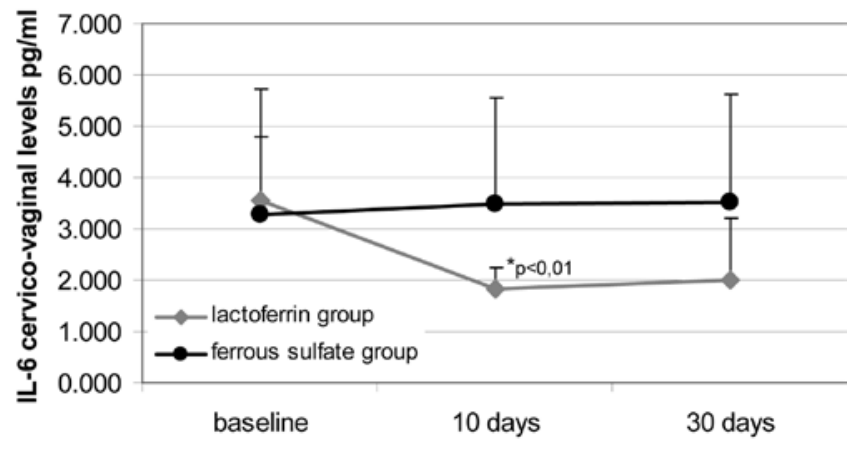

Figure 3. Cervicovaginal IL-6 levels. IL-6 levels detected in the cervicovaginal fluid of female patients treated with either lactoferrin (GA) or ferrous sulphate (GB) at baseline visit, after 10 days and after 30 days of treatment.

with ferrous sulfate (Fig. 2). There were showed elevated levels of pro-inflammatory cytokines, such as IL-6 in the vaginal fluid (IL6cv), in both groups at baseline: IL-6cv mean value $\pm \mathrm{SD}$ was $3.538 \pm 2.2 \mathrm{pg} / \mathrm{ml}$ in the patients treated with Lf and $3.2874 \pm 1.5 \mathrm{pg} / \mathrm{ml}$ in those treated with the ferrous sulfate, with no differences between the two groups ( $\mathrm{p}=0.821$ ) (Fig. 3). It was not possible to determine IL-6 serum (IL6s) levels in 11 patients in the Lf-treated group and in 5 patients from the ferrous sulfate-treated group.

Cervical length values were between 26 and $32 \mathrm{~mm}$ in the female patients included in the Lf group and between 24 and $35 \mathrm{~mm}$ in those included in the placebo group. No funneling was found in any case.
Table I. Clinical characteristics of the female patients enrolled in the study.

\begin{tabular}{lrr}
\hline & $\begin{array}{c}\text { Group A } \\
(\mathrm{N}=14)\end{array}$ & $\begin{array}{c}\text { Group B } \\
(\mathrm{N}=7)\end{array}$ \\
\hline Age & $28 \pm 4$ & $27 \pm 5$ \\
Parity & $1 \pm 1$ & $1 \pm 1$ \\
Days at delivery & $266 \pm 9$ & $258 \pm 4$ \\
Cervical length at baseline (mm) & $29 \pm 2$ & $30 \pm 4$ \\
Cervical length after 30 days (mm) & $28 \pm 2$ & $29 \pm 3$ \\
\hline
\end{tabular}

At first follow-up (10 days) there was no significant change of AVF either in the Lf group or in the ferrous sulfate group (64\% of AVF vs. 57\% of AVF, respectively) (Fig. 2). After 30 days of the Lf treatment, a significant reduction of AVF was observed in the Lf group as compared to those treated with ferrous sulfate (15\% of AVF after 30 days of therapy vs. $57 \%$ of AVF after 30 days in the control group; $\mathrm{p}=0.0007$ ) (Fig. 2).

A decrease in IL-6cv levels was also observed in the patients treated with $\mathrm{Lf}$ as compared to those treated with ferrous sulfate after 10 and 30 days (Fig. 3). After 10 days of therapy the IL-6cv level was $1.834 \pm 0.4 \mathrm{pg} / \mathrm{ml}$ in the female patients taking Lf and $3.466 \pm 1.8 \mathrm{pg} / \mathrm{ml}$ in those taking ferrous sulfate ( $\mathrm{p}=0.005$ ) (Fig. 3). After 30 days the mean IL6cv levels of the two groups were $1.998 \pm 1.2 \mathrm{pg} / \mathrm{ml}$ and $3.532 \pm 2.1 \mathrm{pg} / \mathrm{ml}(\mathrm{p}=0.05)$, respectively (Fig. 3). It was impossible to detect IL-6s levels of the same patients (sensitivity limit, $1.5 \mathrm{pg} / \mathrm{ml}$ ).

The ultrasound data, cervical length and funneling, did not change at follow-up after 10 and 30 days. Pregnancy continued regularly in both groups: All women had TB after 37 weeks (Table I).

\section{Discussion}

Inflammatory cytokines are thought to play an important role in preterm delivery pathogenesis with infection occurring via the uterine cervix $(17,18)$.

Preterm labor and delivery have been associated with elevated amniotic fluid concentrations of IL-6, IL-8 and IL-10. In particular, an elevated concentration of IL- 6 in the amniotic fluid appears to be a marker of intrauterine infection that causes preterm delivery $(19,20)$.

The bacterial endotoxin LPS plays a major role in this process, binding a protein receptor on the macrophages membrane, constituted by the CD14 glycoprotein, TLR4 and the myeloid differentiation protein-2. This causes the activation of myeloid differentiation factor- 88 and other molecules, leading to the activation and nuclear translocation of NF- $\mathrm{KB}$ and the synthesis of inflammatory cytokines, such as IL-1 $\beta$, IL-6 and IL-8, metalloproteinases, glycosaminoglycans, and collagenases, which promote cervical ripening and dilatation (21). Finally, the inflammatory host response promotes cervical ripening and dilatation.

Gram-positive bacteria act through PGN and other bacterial antigens binding TLR2 (3). Such a process leads to PROM and preterm delivery (6-8). Therefore, suppression of the 
inflammatory host response could be crucial in preventing preterm labor and delivery.

In more than $30 \%$ of cases, PROM and preterm delivery are associated with bacterial infection of the amniotic fluid (amnionitis) and elevated rates of inflammatory cytokines, such as IL-6 and IL-8, in the amniotic fluid. This is known as intrauterine inflammatory response syndrome (22). As a result, the fetus may experience fetal inflammatory response syndrome and elevated levels of inflammatory cytokines, such as IL-6 and $\mathrm{TNF} \alpha$ in fetal plasma (22). The latter represents an independent risk factor for severe neonatal morbidity and is strictly associated to neonatal periventricular leukomalacia, developing brain injury due to ischemia in the periventricular area (23).

Lf has a well-known therapeutic indication for combating IDA in pregnant women, as it is able to influence iron homeostasis directly or through other proteins, such as hepcidin, Il-6 or iron transporter ferroportin (FPN), involved in iron transport out of intestinal cells into the blood $(13,14)$. Recent advances on the role of inflammation in iron homeostasis may provide an explanation for the failure of the common therapy with ferrous sulfate in restoring physiological concentrations of total serum iron and serum ferritin.

It is thought that $\mathrm{Lf}$ is capable of protecting against infection through different mechanisms of action, by regulating the iron needed for bacterial proliferation, specifically through its strong iron-binding power $(11,12)$. Lf is also capable of causing cell membrane destruction by binding bacterial cell membrane proteins $(24,25)$. Furthermore, it has an inhibitory effect on LPS-induced production of inflammatory cytokines (TNF- $\alpha$, IL-1 $\beta$, IL-6, and IL-8 mRNA) and in monocytic cells by interfering with $\mathrm{NF}-\kappa \mathrm{B}$ activation $(26,27)$. Additional functions of Lf have been reported, such as neutrophil and macrophage activation (28), regulation of specialization and function of lymphocytes (29), activation of the natural killer cells (30), and control of the oxidation injury (31).

Hasegawa et al have previously demonstrated that LPS-induced IL-6 production in human amnion cells was significantly inhibited by the physiological concentration of Lf in vitro, suggesting that increased levels of Lf under infectious conditions might serve as a self-defense mechanism for both antibacterial and anti-cytokine actions (32). Later, the same research group reported that the transcervical administration of Lf to pregnant rabbits, previously inoculated with $E$. coli, improved fetal survival and prolonged pregnancy (32). Yakuwa and Otsuki conducted an interesting study in preterm pregnant mice uterine cervix modification caused by bacterial vaginal infection, and demonstrated that vaginal administration of rhLf may be a potential as a local treatment for combating host inflammatory response and preventing cervical ripening and dilatation mediated by inflammatory cytokines (16).

The present data suggest that the Lf mechanism of action is to normalize vaginal flora (vaginal infection disappearance) and suppress vaginal inflammatory response (reduction of IL-6 levels on cervicovaginal fluid).

We found that oral administration of $100 \mathrm{mg}$ Lf twice a day for one month, causes reduction of IL- 6 in cervicovaginal fluid (IL6cv) and the normalization of vaginal flora in women at risk of preterm delivery. Apart from the clinical data and the lack of effects on cervical parameter at TV-US, our study suggests that Lf prolongs gestation by suppressing the IL-6 cervicovaginal fluid level in preterm pregnant women. These preliminary data are consistent with the preclinical data on the role of Lf as a down-modulator of ongoing immuneinflammatory responses during preterm delivery.

Further studies are warranted to confirm our data which provide valuable proof of concept for the potential use of Lf for the prevention of preterm delivery.

\section{References}

1. Randis TM: Progress toward understanding of infection-related preterm birth. Clin Perinatol 37: 677-688, 2010.

2. Donders GG: Definition and classification of abnormal vaginal flora. Best Pract Res Clin Obstet Gynaecol 21: 355-373, 2007

3. Chiu YC, Lin CY, Chen CP, et al: Peptidoglycan enhances IL-6 production in human synovial fibroblasts via TLR2 receptor, focal adhesion kinase, Akt, and AP-1 dependent pathway. J Immunol 183: 2785-2792, 2009.

4. Elass E, Masson M, Mazurier J and Legrand D: Lactoferrin inhibits the lipopolysaccharide-induced expression and proteoglycanbinding ability of interleukin- 8 in human endothelial cells Infect Immun 70: 1860-1866, 2002.

5. Liggins GC: Cervical ripening as an inflammatory reaction. In: The cervix in Pregnancy and Labor.Ellwood DA, Anderson ABM (eds). Churchill-Livingstone, Edinburgh, pp1-9, 1983.

6. Winkler M: Role of cytokines and other inflammatory mediators. Br J Obstet Gynecol 110 (Suppl 20): 118-123, 2003.

7. Sakai M, Sasaki Y, Yoneda S, et al: Elevated interleukin-8 in cervical mucus as an indicator for treatment to prevent premature birth and preterm, pre-labor rupture of membranes: a prospective study. Am J Reprod Immunol 51: 220-225, 2004.

8. Keelan JA, Blumenstein M, Helliwell RJ, et al: Cytokines, prostaglandins, and parturitionda review. Placenta 24 (Suppl A): S33-S46, 2003.

9. Rodríguez-Franco DA, Vázquez-Moreno L and Ramos-Clamont Montfort G: Antimicrobial mechanisms and potential clinical application of lactoferrin. Rev Latinoam Microbiol 47: 102-111, 2005.

10. Arnold RR, Brewer M and Gauthier JJ: Bactericidal activity of human lactoferrin: sensitivity of a variety of microorganisms. Infect Immun 28: 893-898, 1980.

11. Staut J, Norrell S and Harrington JP: Kinetic effect of human lactoferrin on growth of Escherichia coli O-111. Int J Biochem 16: 1043-1047, 1984

12. Ellison RT III, Giehl TJ and LaForce FM: Damage of outer membrane of the enteric Gram-negative bacteria by lactoferrin and transferring. Infect Immun 56: 2774-2781, 1988.

13. Paesano R, Pietropaoli M, Gessani S and Valenti P: The influence of lactoferrin, orally admnistered, on systemic iron homeostasis in pregnant women suffering of iron deficiency and iron deficiency anemia. Biochimie 91: 44-51, 2009.

14. Paesano R and Torcia F, Berlutti F, et al: Oral administration of lactoferrin increases hemoglobin and total serum iron in pregnant women. Biochem Cell Biol 84: 377-380, 2006.

15. Bothwell $\mathrm{H}$ : Iron requirements in pregnancy and strategies to meet them. Am J Clin Nutr 72: S257-S264, 2000.

16. Yakuwa K and Otsuki K: Recombinanat human lactoferrin has a potential to suppress uterine cervical ripening in preterm delivery in animal model. Arch Gynecol Obtet 275: 331-334, 2007.

17. Wei SQ, Fraser W and Luo ZC: Inflammatory cytokines and spontaneous preterm birth in asymptomatic women: a systematic review. Obstet Gynecol 116 (2 Pt 1): 393-401, 2010.

18. Kemp MW, Saito M, Newnham JP, et al: Preterm birth, infection, and inflammation advances from the study of animal models. Reprod Sci 17: 619-628, 2010.

19. Lange M, Chen FK, Wessel J, Buscher U and Dudenhausen JW: Elevation of interleukin-6 levels in cervical secretions as a predictor of preterm delivery. Acta Obstet Gynecol Scand 82: 326-329, 2003.

20. Romero R, Yoon BH, Mazor M, et al: The diagnostic and prognostic value of amniotic fluid white blood cell count, glucose, interleukin-6, and Gram stain in patients with preterm labor and intact membranes. Am J Obstet Gynecol 169: 805-816, 1993.

21. Mitsuhiro F, Masashi M, Tanamoto K, Suzuki T, Azuma H and Ikeda H: Molecular mechanisms of macrophage activation and deactivation by lipopolysaccharide: roles of the receptor complex. Pharmacol Ther 100: 171-194, 2003. 
22. Dudley DJ: Pre-term labor: an intra-uterine inflammatory response syndrome? J Reprod Immunol 36: 93-109, 1997.

23. Yoon BH,Park CW and Chaiworapongsa T: Intrauterine infection and the development of cerebral palsy. Br J Obstet Gynecol 20 (Suppl 110): 124-127, 2003.

24. Soukka T, Tenovuo J and Lenander-Lumikari M: Fungicidal effect of human lactoferrin against Candida albicans. FEMS Microbiol Lett 90: 223-228, 1992.

25. Naidu SS, Svensson U, Kishore AR and Naidu AS: Relationship between antibacterial activity and porin binding of lactoferrin in Escherichia coli and Salmonella typhimurium. Antimicrob Agents Chemother 37: 240-256, 1993.

26. Haversen L, Ohlsson GB, Hahn-Zoric M, Hanson LA and Mattsby-Baltzer I: Lactoferrin down-regulates the LPS-induced production in monocytic cells via NF-kB. Cell Immunol 220: 83-95, 2002.

27. Crouch SPM, Slater KJ and Fletcher J: Regulation of cytokine release from mononuclear cells by the iron-binding protein lactoferrin. Blood 80: 235-240, 1992.
28. Lima MF and Kierszenbaum F: Lactoferrin effects on phagocytic cell function increased uptake and killing of an intracellular parasite by murine macrophages and monocytes. J Immunol 134: 4176-4183, 1985.

29. Zimecki M, Mazurier J, Machnicki MZ, Machnicki M, Wieczorek Z, Montreuil J and Spik G: Immunostimulatory activity of lactotransferrin and maturation of CD4, CD8 murine thymocytes. Immunol Lett 30: 119-124, 1991.

30. Shau H, Kim A and Golub SH: Modulation of natural killer and lymphokine-activated killer cell cytotoxicity by lactoferrin. J Leuk Biol 51: 343-349, 1992.

31. Cohen MS, Mao J, Rasmussen GT, Rasmussen GT, Serody JS and Britigan BE: Interaction of lactoferrin and lipopolysaccharide: effects on the antioxidant property of lactoferrin and the ability of lipopolysaccharide to prime human neutrophils for enhanced superoxide formation. J Infect Dis 166: 1375-1378, 1992.

32. Hasegawa A, Otsuki K, Sasaki Y, et al: Preventive effect of recombinant human lactoferrin in a rabbit pre-term delivery model. Am J Obstet Gynecol: 1038-1043, 2005. 